\title{
An Assessment of the Diabetic Knowledge, Attitude, and Practice of School Teachers in Riyadh, Kingdom of Saudi Arabia
}

\author{
Ghassan Aldekhayel (1) \\ Diabetes Care Center, King Salman Hospital, Riyadh, Saudi Arabia \\ Email: dr.gass@hotmail.com
}

How to cite this paper: Aldekhayel, G. (2020) An Assessment of the Diabetic Knowledge, Attitude, and Practice of School Teachers in Riyadh, Kingdom of Saudi Arabia. Journal of Diabetes Mellitus, 10, 132-153.

https://doi.org/10.4236/jdm.2020.103012

Received: June 6, 2020

Accepted: August 7, 2020

Published: August 10, 2020

Copyright $\odot 2020$ by author(s) and Scientific Research Publishing Inc. This work is licensed under the Creative Commons Attribution International License (CC BY 4.0).

http://creativecommons.org/licenses/by/4.0/

\begin{abstract}
Background: Teachers are the cornerstone of the next generation and they are considered an important source of health information for students about diabetes. The objective of this study is to assess the knowledge, attitude, and practice (KAP) of teachers about diabetes mellitus (DM) and to identify the associated factors. Methods: Cross-sectional study design included a stratified random sample of male and female teachers from public and private schools in Riyadh city. A self-administered online questionnaire was developed. The data collection was from October to November 2019. Results: The study included 633 teachers. Overall, $57.2 \%$ of the surveyed teachers were knowledgeable about DM. Teachers aged between 51 and 60 years $(p=0.005)$, males $(\mathrm{p}=0.018), \mathrm{PhD}$ holders $(\mathrm{p}=0.011)$, teaching science $(\mathrm{p}=0.021)$, having more than 20 years of teaching experience $(\mathrm{p}=0.001)$, diabetics $(\mathrm{p}<0.001)$, and having family history of diabetes $(\mathrm{p}=0.007)$ had the highest level of knowledge. Overall, positive attitude towards DM was reported among 53.1\% of the teachers. Those with teaching experience between 11 and 15 years ( $\mathrm{p}=$ $0.024)$, diabetics $(p=0.029)$, and having a diabetic student $(p=0.012)$ had more positive attitude towards diabetes. Good diabetes-related practice was observed among $54.3 \%$ of the teachers. Older $(\mathrm{p}<0.001)$, having more than 20 years of teaching experience $(\mathrm{p}<0.001)$, non-Saudi $(\mathrm{p}=0.011)$, diabetic teachers with longer duration of the disease $(\mathrm{p}<0.001)$, having family history of DM ( $p=0.028)$, and principals $(\mathrm{p}=0.002)$ had the highest level of good diabetes-related practice. Conclusion: The KAP of teachers in public and private schools in Riyadh about DM was sufficient in many aspects. However, targeting new young teachers should be a priority to raise awareness.
\end{abstract}

\section{Keywords}

Knowledge, Attitude, Practice, KAP, Awareness, Diabetes Mellitus, Saudi Arabia 


\section{Introduction}

Diabetes is one of the most common non-communicable diseases worldwide. There are three main types of diabetes type 1, type 2 , and gestational diabetes. Type 1 diabetes accounts for 5\% - 10\% of diabetes and mostly affects children and adolescents. Meanwhile, type 2 diabetes accounts for $90 \%$ - 95\% of all diabetes. Most patients with type 2 diabetes are overweight or obese, because of their sedentary lifestyle, high calorie intake, also body fat itself causes insulin resistance. In both types of diabetes type 1 and type 2, genetic and environmental factors cause the loss of beta cells in the pancreas resulting in hyperglycemia [1].

Over the past 30 years the incidence rate of type 1 diabetes has increased in the Kingdom of Saudi Arabia (KSA) [2]. According to the most recent International Diabetes Federation (IDF) report in 2019, KSA is one of the top ten countries worldwide for the number of incidence per year of type 1 diabetes in children and adolescents aged 0 - 14 years. According to the IDF report KSA is the fifth country worldwide (after Finland, Sweden, Kuwait, and Norway) in the incidence rates per 100,000 population per year of type 1 diabetic children and adolescents aged 0 - 14 years with an incidence rate of 31.4 per 100,000 population per year. The IDF also reported that in the Middle East and North Africa (MENA) Region KSA is the third country (after Algeria and Morocco) for the number of children and adolescents aged 0 - 19 years with type 1 diabetes with an estimated number of 27,800 diabetic children and adolescent [3].

During the school day type 1 diabetic children and adolescents need to check their blood glucose levels, have a certain diet and receive their insulin treatment thus the American Diabetes Association (ADA) recommends that school teachers be knowledgeable to provide proper care for diabetic students and be trained to manage diabetic emergencies [4] [5].

Thus it is important to raise the awareness level of school teachers about type 1 diabetes through health education campaigns in order to lower the risk of $\mathrm{Di}$ abetic ketoacidosis (DKA) among type 1 diabetic students [6]. Therefore increasing teachers' knowledge about diabetes will have positive effects on ensuring diabetic students' safety in school.

In 2004, a nationwide study revealed the magnitude of type 2 diabetes as the results of the study showed that $23.7 \%$ of Saudi people are diabetic and $27.9 \%$ of them are unaware of having the disease [7]. A more recent nationwide study done in 2015 , called out for an urgent primary prevention program for diabetes. The study showed that the prevalence of diabetes is $25.4 \%$, meanwhile $25.5 \%$ have impaired fasting glucose, so about half of the Saudi population are either diabetic or at high risk of developing diabetes [8].

Diabetes has a high financial cost on a nation's healthcare system, because it is a chronic disease that causes many complications later in a patient's life. The healthcare expenditures not only go toward the treatment of diabetes itself, but also the treatment of its lifelong associated complications. The economic burden of diabetes on the MENA Region in 2019 was approximately 24.9 billion United 
States dollars (USD). In 2020, the high prevalence of diabetes in KSA is projected to cost the Ministry of Health 7.4 billion USD [3] [9].

Public health has evolved from both managing the acute symptoms of diabetes and preventing its long term complications, towards additional means concentrating on the primary prevention of diabetes. So countries with high prevalence of diabetes should urgently implement a public health approach to diabetes mellitus (DM) prevention, through increasing the level of health awareness among their communities [10].

Implementing a public health approach to diabetes prevention in a specific group of the community such as school teachers, requires assessing their Knowledge, attitude, and practice (KAP) and identifying the associated factors. Most studies assessing KAP in KSA have been done on diabetic patients and some on non-diabetic patients [11] [12].

There are very limited studies that have assessed KAP on type 1 diabetes among school teachers in KSA. One study was done in Al-jouf district public schools, in the Northern part of KSA [2]. Another study was done in Al Baha city public schools in the Southern part of KSA [13], and a study was done in Makah in the Western part of KSA that only assessed the awareness of school teachers [14]. Even less publications assessed KAP of type 2 diabetes among school teachers in KSA. A study assessed the knowledge among self-reported diabetic female teachers in Al-Khobar city, in the Eastern part of KSA [15]. However, none of the studies done in KSA have assessed KAP for both type 1 and type 2 diabetes among school teachers in Riyadh city.

Riyadh city is the capital of KSA and is home for almost 6.5 million people [16]. Most Saudis living in the capital city have migrated with their families from all parts of KSA to work in the public or the private sector. Moreover, many non-Saudi individuals and families moved to Riyadh city from different parts of the world especially Muslim and Arab countries to work and study. The different backgrounds of the population living in Riyadh makes it special for conducting research, because the results can be generalized nationwide. Therefore, the findings of this study would have significance in developing a specific and target-oriented health education program for school teachers in KSA.

\section{Methods}

This research study was designed as a quantitative cross sectional study. A stratified sampling technique was applied for randomization. For the purpose of this study, the population included are all teachers from schools in Riyadh city. According to the Ministry of Education Statistics there are 79,188 male and female teachers working at public and private schools in Riyadh city throughout the school year 2019-2020 [17]. The Sample size needed for the study population was 383. The Sample size was calculated using OpenEpi [18], which is an open source sample size calculator for public health. A total of 633 male and female teachers working at public and private schools participated in this study. Partic- 
ipants were randomized by means of stratified sampling technique by the Technology Department at the Ministry of Education using their database. The online questionnaire link was send via short message service (SMS) to the randomized sample of male and female teachers working at public and private schools in Riyadh city. The response rate for the online questionnaire was $6.33 \%$. The data collection period was for one month from 16 October 2019.

\subsection{Research Tool}

The research tool used in this study was a self-administered online questionnaire in Arabic language, which was developed after thoroughly reviewing relevant literature [5] [11] [19] [20] [21]. The questionnaire was approved by the Institutional review board (IRB) committee of King Saud University and the Department of Planning and Development at the Ministry of Education.

The questionnaire consisted of four parts. The first part was the personal details section which included 9 questions about the demographics of the participants. The second part of the questionnaire consisted of 12 questions, which assessed the knowledge of participants. Part three of the questionnaire had 7 questions that assessed the attitude of participants toward diabetes. The fourth and final part contained 15 questions that assessed diabetes related practices of the participants. The questionnaire was developed in Arabic language to allow the participants to answer in their first language, then it was translated to English language for the purpose of data analysis. The questionnaire was piloted on a sample of 46 teachers and was not included in the data analysis for this study.

\subsection{Reliability}

The questionnaire was carefully developed to not include any leading questions. The order of the questions was designed to not influence the participants' answers to following questions. The time needed to complete the questionnaire was around 5 to 7 minutes to avoid fatigue of the participants. The questionnaire was developed in Arabic language to allow the participants to answer in their first language, then it was translated to English language (Appendix) for the purpose of data analysis.

\subsection{Validity}

To insure the validity of the questionnaire, the research team developed the questionnaire after thoroughly reviewing relevant literature [5] [11] [19] [20] [21]. A pilot study was done to ensure that the questionnaire was easily understood by the participants.

\subsection{Statistical Analysis}

The data was analyzed using SPSS 25.0 version statistical software. Descriptive statistics (frequencies and percentages) were used to describe the categorical variables. Knowledge, attitude and practice responses were given 1 point for each correct/favorable answer, while the incorrect/unfavorable/unknown answer was 
given zero point. Classification of participants was done using mean scores of participants' responses.

Some of the knowledge section questions were used as diabetes related characteristics of the participants as factors associated with participants' knowledge, attitude and practice. These questions include the source of information question (question 1) and the diabetes related data questions such as questions 2 - 5, 11 , and 12 .

The rest of the knowledge section questions were calculated as the sum of correct answers. There were two types of questions in the knowledge section, questions having two possible answers such as questions 6,7 , and 10, which were given 1 point for the correct answer and zero point for the incorrect or unknown answer. The other type of questions had multiple correct answers such as question 8 had 5 correct answers and question 9 had 7 correct answers, thus 1 point was given for each correct answer and zero point for the incorrect or unknown answer. Therefore, knowledge total scores range between 0 and 15 .

Attitude and practice scores were calculated as the sum of favorable responses. The attitude and practice sections had one type of questions with two possible answers, the favorable responses were given 1 point, while the unfavorable or unknown responses were given zero point. Thus attitude total scores range between 0 and 7, meanwhile practice total scores range between 0 and 15 .

Mean scores of participants' responses were calculated for each of the knowledge, attitude and practice sections. The mean scores of the participants' responses were used as cut-off scores to classify the participants. As result, the participants' knowledge was classified to (not knowledgeable and knowledgeable), attitude was classified to (negative and positive) and practice was classified to (poor and good) [22]. Pearson's chi-square test was used for bivariate statistical analysis. Meanwhile, Chi-square for trend test was used for the statistical analysis of ordinal variable. p-value of 0.05 was used as the cut-off for statistical significance of the results.

\section{Results}

The study included 633 participants, their personal characteristics are summarized in Table 1 . The age of $60 \%$ of participants was over 40 years old, as well as males represented $52.3 \%$ of them. Most of the participants (84.7\%) had a bachelor degree, also the most frequent majors were Arabic language (22.1\%) and Islamic studies $(21.3 \%)$. The majority of the participants (86\%) were teachers and almost two-thirds (64.6\%) of them worked at public schools. Additionally, over one-third of the participants (41.6\%) had more than 20 years of teaching experience. Finally, the majority of participants were married (88.8\%) and Saudi (85.8\%).

The most frequent sources of information about DM were relatives/friends (58.6\%), social media $(41.4 \%)$, internet $(31.1 \%)$, physicians $(24 \%)$, and awareness campaigns (22.1\%) (Table 2). 
The prevalence of DM among participants was $17.7 \%$. In $42.8 \%$ of diabetic teachers the duration of DM ranged between 1 to 5 years. Most teachers (74.7\%) had a family history of DM, mostly among parents (72.6\%). Less than one-third of teachers (30\%) reported the presence of glucometer at school, meanwhile $59.1 \%$ had a diabetic student (Table 3 ).

Table 1. Personal characteristics of the participants $(n=633)$.

\begin{tabular}{|c|c|c|c|}
\hline & Categories & Frequency $\mathrm{N}$ & Percentage \% \\
\hline \multirow{4}{*}{ Age (years) } & $20-30$ & 49 & 7.7 \\
\hline & $31-40$ & 204 & 32.3 \\
\hline & $41-50$ & 249 & 39.3 \\
\hline & $51-60$ & 131 & 20.7 \\
\hline \multirow{2}{*}{ Gender } & Male & 331 & 52.3 \\
\hline & Female & 302 & 47.7 \\
\hline \multirow{4}{*}{ Qualification } & Diploma & 42 & 6.6 \\
\hline & Bachelor & 536 & 84.7 \\
\hline & Master & 48 & 7.6 \\
\hline & $\mathrm{PhD}$ & 7 & 1.1 \\
\hline \multirow{8}{*}{ Major } & Mathematics & 75 & 11.8 \\
\hline & Science & 79 & 12.5 \\
\hline & Islamic studies & 134 & 21.3 \\
\hline & Art education & 45 & 7.1 \\
\hline & Social studies & 83 & 13.1 \\
\hline & Arabic language & 140 & 22.1 \\
\hline & English language & 54 & 8.5 \\
\hline & Physical education & 23 & 3.6 \\
\hline \multirow{4}{*}{ Professional title } & Principal & 30 & 4.7 \\
\hline & Deputy principal & 33 & 5.2 \\
\hline & Teacher & 544 & 86.0 \\
\hline & Student counselor & 26 & 4.1 \\
\hline \multirow{2}{*}{ Type of school } & Public & 409 & 64.6 \\
\hline & Private & 224 & 35.4 \\
\hline \multirow{5}{*}{ Years of experience } & $1-5$ & 66 & 10.4 \\
\hline & $6-10$ & 121 & 19.1 \\
\hline & $11-15$ & 79 & 12.5 \\
\hline & $16-20$ & 104 & 16.4 \\
\hline & $>20$ & 263 & 41.6 \\
\hline \multirow{2}{*}{ Marital status } & Single & 71 & 11.2 \\
\hline & Married & 562 & 88.8 \\
\hline \multirow{2}{*}{ Nationality } & Saudi & 543 & 85.8 \\
\hline & Non-Saudi & 90 & 14.2 \\
\hline
\end{tabular}


Table 2. Source of information about diabetes mellitus among the participants.

\begin{tabular}{ccc}
\hline & Frequency N & Percentage \% \\
\hline Relatives/friends & 371 & 58.6 \\
Social media & 262 & 41.4 \\
Medical book/scientific journal & 80 & 12.6 \\
Search on the Internet & 197 & 31.1 \\
Health educator & 107 & 16.9 \\
Physician & 152 & 24.0 \\
Awareness campaign about diabetes & 140 & 22.1 \\
\hline
\end{tabular}

Table 3. Diabetes-related characteristics of the participants.

\begin{tabular}{|c|c|c|}
\hline & Frequency $\mathbf{N}$ & Percentage \% \\
\hline \multicolumn{3}{|c|}{ Personal history of diabetes mellitus } \\
\hline Yes & 112 & 17.7 \\
\hline No & 521 & 82.3 \\
\hline \multicolumn{3}{|c|}{ Family history of diabetes mellitus } \\
\hline Yes & 473 & 74.7 \\
\hline No & 160 & 25.3 \\
\hline \multicolumn{3}{|c|}{ Family members diagnosed with diabetes mellitus $(n=470)$} \\
\hline Parents & 341 & 72.6 \\
\hline Spouse & 39 & 8.3 \\
\hline Brother/sister & 74 & 15.7 \\
\hline Son/Daughter & 16 & 3.4 \\
\hline \multicolumn{3}{|c|}{ Duration of diabetes in years $(\mathrm{n}=112)$} \\
\hline$<1$ & 14 & 12.5 \\
\hline $1-5$ & 48 & 42.8 \\
\hline $6-10$ & 27 & 24.1 \\
\hline $11-15$ & 12 & 10.7 \\
\hline $16-20$ & 5 & 4.5 \\
\hline$>20$ & 6 & 5.4 \\
\hline \multicolumn{3}{|c|}{ Presence of Glucometer at school } \\
\hline Yes & 190 & 30.0 \\
\hline No & 443 & 70.0 \\
\hline \multicolumn{3}{|c|}{ Having a diabetic student at class } \\
\hline Yes & 374 & 59.1 \\
\hline No & 259 & 40.9 \\
\hline
\end{tabular}

\subsection{Knowledge about Diabetes Mellitus}

Overall, $57.2 \%$ of the surveyed teachers were knowledgeable about DM. The majority of participants knew that diabetes can be prevented through sports and eating healthy (98.1\%), also most of them knew that Pancreas dysfunction leads 
to diabetes (96.1\%). However, only $25.8 \%$ recognized the fasting plasma glucose cut-off level to diagnose diabetes. Regarding diabetes complications, the most known complication was retinopathy $(76.8 \%)$ and the least known was stroke (18.6\%). Obesity was the most known risk factor (85.5\%) among participants, while the least known was smoking (27.3\%).

Factors associated with knowledge about diabetes mellitus are shown in Table 4. Almost two-thirds (65.6\%) of teachers aged 51 to 60 years compared to $44.9 \%$ of teachers aged 20 to 30 years were knowledgeable about DM, p $=0.005$. Male teachers were more knowledgeable compared to females (61.6\% versus $52.3 \%$ ), p $=0.018$. Most teachers with a $\mathrm{PhD}$ degree $(71.4 \%)$ compared to $42.9 \%$ of Diploma holders were knowledgeable, $\mathrm{p}=0.011$. The highest rate of knowledge regarding DM was reported among science teachers (68.4\%) whereas the lowest rate was observed among social studies teachers (47\%), $\mathrm{p}=0.021$. Nearly two-thirds $(64.6 \%)$ of teachers with more than 20 years of experience compared to only $47 \%$ of those with an experience of 1 to 5 years were knowledgeable, $p=0.001$. Diabetic teachers were more knowledgeable about DM compared to non-diabetics (76.8\% versus 53\%), $\mathrm{p}<0.001$. Also, teachers with a family history of diabetes were more knowledgeable than teachers with no family history of diabetes $(60.3 \%$ versus $48.1 \%), p=0.007$. Finally, teachers who reported that their source of information about DM was medical book/scientific journal were more knowledgeable compared to teachers who reported other sources of information, $\mathrm{p}<0.001$.

\subsection{Attitude towards Diabetes Mellitus}

Overall, positive attitude towards diabetes was reported among 53.1\% of teachers. The majority of teachers (92.9\%) thought that attending awareness and educational lectures about diabetes will help improve a diabetic patient and $91.8 \%$ were willing to accept diabetic students in their class. Three quarters of teachers did not think that it is useless to try to control blood glucose because complications of diabetes will occur anyway. However, almost $40 \%$ of teachers thought that sports are better to treat diabetes than taking prescription medications. Also, only $18.8 \%$ reported not feeling anxious when first informed to have children with diabetes in their class.

Factors associated with attitude towards diabetes mellitus are shown in Table 5. We can see that the highest rate of positive attitude towards DM was among teachers with 11 to 15 years of experience (62\%), whereas the lowest rate of positive attitude was among teachers with 1 to 5 years of experience $(37.9 \%), p=0.024$. Teachers that are diabetic for more than 20 years had a higher rate of positive attitude towards diabetes compared to teachers with less than one year history of DM (83.3\% versus $28.6 \%$ ), however the difference was borderline insignificant, $\mathrm{p}$ $=0.053$. Teachers with a family history of DM expressed more positive attitude towards the disease than those without such a history (55.6\% versus $45.6 \%), \mathrm{p}=$ 0.029. Furthermore, teachers who had a diabetic student showed more positive attitude towards DM than their counterparts (57.2\% versus $47.1 \%), \mathrm{p}=0.012$. 
Table 4. Factors associated with teachers' knowledge about diabetes mellitus.

\begin{tabular}{|c|c|c|c|}
\hline \multirow[b]{2}{*}{ Variables } & \multicolumn{2}{|c|}{ Diabetes Mellitus Knowledge level } & \multirow[b]{2}{*}{ p-value } \\
\hline & $\begin{array}{c}\text { Not Knowledgeable } \\
\mathrm{N}=271 \\
\mathrm{~N}(\%)\end{array}$ & $\begin{array}{c}\text { Knowledgeable } \\
\mathrm{N}=362 \\
\mathrm{~N}(\%)\end{array}$ & \\
\hline \multicolumn{4}{|l|}{ Age } \\
\hline $20-30(n=49)$ & $27(55.1)$ & $22(44.9)$ & \\
\hline $31-40(n=204)$ & $95(46.6)$ & $109(53.4)$ & \\
\hline $41-50(n=249)$ & $104(41.8)$ & $145(58.2)$ & \\
\hline $51-60(n=131)$ & $45(34.4)$ & $86(65.6)$ & $0.005^{\mathrm{b}}$ \\
\hline \multicolumn{4}{|l|}{ Gender } \\
\hline Male $(\mathrm{n}=331)$ & $127(38.4)$ & $204(61.6)$ & \\
\hline Female $(\mathrm{n}=302)$ & $144(47.7)$ & $158(52.3)$ & $0.018^{\mathrm{a}}$ \\
\hline \multicolumn{4}{|l|}{ Qualification } \\
\hline Diploma $(n=42)$ & $24(57.1)$ & $18(42.9)$ & \\
\hline Bachelor $(n=536)$ & $230(42.9)$ & $306(57.1)$ & \\
\hline Master $(n=48)$ & $15(31.3)$ & $33(68.7)$ & \\
\hline $\mathrm{PhD}(\mathrm{n}=7)$ & $2(28.6)$ & $5(71.4)$ & $0.011^{\mathrm{b}}$ \\
\hline \multicolumn{4}{|l|}{ Major } \\
\hline Mathematics $(\mathrm{n}=75)$ & $26(34.7)$ & $49(65.3)$ & \\
\hline Science $(\mathrm{n}=79)$ & $25(31.6)$ & $54(68.4)$ & \\
\hline Islamic studies $(\mathrm{n}=134)$ & $60(44.8)$ & $74(55.2)$ & \\
\hline Art education $(\mathrm{n}=45)$ & $16(35.6)$ & $29(64.4)$ & \\
\hline Social studies $(\mathrm{n}=83)$ & $44(53.0)$ & $39(47.0)$ & \\
\hline Arabic language $(\mathrm{n}=140)$ & $71(50.7)$ & $69(49.3)$ & \\
\hline English language $(\mathrm{n}=54)$ & $18(33.3)$ & $36(66.7)$ & \\
\hline Physical education $(\mathrm{n}=23)$ & $11(47.8)$ & $12(52.2)$ & $0.021^{\mathrm{a}}$ \\
\hline \multicolumn{4}{|l|}{ Professional title } \\
\hline Principal $(\mathrm{n}=30)$ & $11(36.7)$ & $19(63.3)$ & \\
\hline Deputy principal $(\mathrm{n}=33)$ & $14(42.4)$ & $19(57.6)$ & \\
\hline Teacher $(\mathrm{n}=544)$ & $238(43.8)$ & $306(56.3)$ & \\
\hline Student counselor $(n=26)$ & $8(30.8)$ & $18(69.2)$ & $0.532^{\mathrm{a}}$ \\
\hline \multicolumn{4}{|l|}{ Type of school } \\
\hline Public $(\mathrm{n}=409)$ & $166(40.6)$ & $243(59.4)$ & \\
\hline Private $(\mathrm{n}=224)$ & $105(46.9)$ & $119(53.1)$ & $0.126^{\mathrm{a}}$ \\
\hline \multicolumn{4}{|l|}{ Years of experience } \\
\hline $1-5(n=66)$ & $35(53.0)$ & $31(47.0)$ & \\
\hline $6-10(n=121)$ & $62(51.2)$ & $59(48.8)$ & \\
\hline $11-15(\mathrm{n}=79)$ & $31(39.2)$ & $48(60.8)$ & \\
\hline $16-20(\mathrm{n}=104)$ & $50(48.1)$ & $54(51.9)$ & \\
\hline$>20(\mathrm{n}=263)$ & $93(35.4)$ & $170(64.6)$ & $0.001^{\mathrm{b}}$ \\
\hline \multicolumn{4}{|l|}{ Marital status } \\
\hline Single $(\mathrm{n}=71)$ & $35(49.3)$ & $36(50.7)$ & \\
\hline Married $(\mathrm{n}=562)$ & $236(42.0)$ & $326(58.0)$ & $0.241^{\mathrm{a}}$ \\
\hline \multicolumn{4}{|l|}{ Nationality } \\
\hline Saudi $(\mathrm{n}=543)$ & $238(43.8)$ & $305(56.2)$ & \\
\hline Non - Saudi $(\mathrm{n}=90)$ & $33(36.7)$ & $57(63.3)$ & $0.203^{\mathrm{a}}$ \\
\hline
\end{tabular}

aearson's chi-square; ${ }^{\text {b }} \mathrm{Chi}$-square for trend. 
Table 5. Factors associated with teachers' attitude towards diabetes mellitus.

\begin{tabular}{|c|c|c|c|}
\hline \multirow[b]{2}{*}{ Variables } & \multicolumn{2}{|c|}{ Attitude towards Diabetes Mellitus } & \multirow[b]{2}{*}{$\mathrm{p}$-value } \\
\hline & $\begin{array}{c}\text { Negative }(\mathrm{N}=297) \\
\text { N (\%) }\end{array}$ & $\begin{array}{c}\text { Positive }(\mathrm{N}=336) \\
\mathrm{N}(\%)\end{array}$ & \\
\hline \multicolumn{4}{|l|}{ Age } \\
\hline $20-30(n=49)$ & $28(57.1)$ & $21(42.9)$ & \\
\hline $31-40(\mathrm{n}=204)$ & $94(46.1)$ & $110(53.9)$ & \\
\hline $41-50(\mathrm{n}=249)$ & $113(45.4)$ & $136(54.6)$ & \\
\hline $51-60(n=131)$ & $62(47.3)$ & $69(52.7)$ & $0.480^{\mathrm{b}}$ \\
\hline \multicolumn{4}{|l|}{ Gender } \\
\hline Male $(\mathrm{n}=331)$ & $158(47.7)$ & $173(52.3)$ & \\
\hline Female $(\mathrm{n}=302)$ & $139(46.0)$ & $163(54.0)$ & $0.667^{\mathrm{a}}$ \\
\hline \multicolumn{4}{|l|}{ Qualification } \\
\hline Diploma $(\mathrm{n}=42)$ & $26(61.9)$ & $16(38.1)$ & \\
\hline Bachelor $(\mathrm{n}=536)$ & $247(46.1)$ & $289(53.9)$ & \\
\hline Master $(\mathrm{n}=48)$ & 19 (39.6) & $29(60.4)$ & \\
\hline $\mathrm{PhD}(\mathrm{n}=7)$ & $5(71.4)$ & $2(28.6)$ & $0.238^{\mathrm{b}}$ \\
\hline \multicolumn{4}{|l|}{ Major } \\
\hline Mathematics $(\mathrm{n}=75)$ & $38(50.7)$ & $37(49.3)$ & \\
\hline Science $(\mathrm{n}=79)$ & $27(34.2)$ & $52(65.8)$ & \\
\hline Islamic studies $(\mathrm{n}=134)$ & $70(52.2)$ & $64(47.8)$ & \\
\hline Art education $(\mathrm{n}=45)$ & $18(40.0)$ & $27(60.0)$ & \\
\hline Social studies $(\mathrm{n}=83)$ & $42(50.6)$ & $41(49.4)$ & \\
\hline Arabic language $(\mathrm{n}=140)$ & $70(50.0)$ & $70(50.0)$ & \\
\hline English language $(\mathrm{n}=54)$ & $24(44.4)$ & $30(55.6)$ & \\
\hline Physical education $(\mathrm{n}=23)$ & $8(34.8)$ & $15(65.2)$ & $0.165^{\mathrm{a}}$ \\
\hline \multicolumn{4}{|l|}{ Professional title } \\
\hline Principal $(\mathrm{n}=30)$ & $13(43.3)$ & $17(56.7)$ & \\
\hline Deputy principal $(\mathrm{n}=33)$ & $13(39.4)$ & $20(60.6)$ & \\
\hline Teacher $(\mathrm{n}=544)$ & $257(47.2)$ & $287(52.8)$ & \\
\hline Student counselor $(n=26)$ & $14(53.8)$ & $12(46.2)$ & $0.699^{\mathrm{a}}$ \\
\hline \multicolumn{4}{|l|}{ Type of school } \\
\hline Public $(n=409)$ & $198(47.4)$ & $215(52.6)$ & \\
\hline Private $(\mathrm{n}=224)$ & $103(46.0)$ & $121(54.0)$ & $0.727^{\mathrm{a}}$ \\
\hline \multicolumn{4}{|l|}{ Years of experience } \\
\hline $1-5(n=66)$ & $41(62.1)$ & $25(37.9)$ & \\
\hline $6-10(\mathrm{n}=121)$ & $59(48.8)$ & $62(51.2)$ & \\
\hline $11-15(n=79)$ & $30(38.0)$ & $49(62.0)$ & \\
\hline $16-20(\mathrm{n}=104)$ & $41(39.4)$ & $63(60.6)$ & \\
\hline$>20(\mathrm{n}=263)$ & $126(47.9)$ & $137(52.1)$ & $0.024^{\mathrm{b}}$ \\
\hline \multicolumn{4}{|l|}{ Marital status } \\
\hline Single $(\mathrm{n}=71)$ & $37(52.1)$ & $34(47.9)$ & \\
\hline Married $(n=562)$ & $260(46.3)$ & $302(53.7)$ & $0.352^{\mathrm{a}}$ \\
\hline \multicolumn{4}{|l|}{ Nationality } \\
\hline Saudi $(\mathrm{n}=543)$ & $252(46.4)$ & $291(53.6)$ & \\
\hline Non - Saudi $(\mathrm{n}=90)$ & $45(50.0)$ & $45(50.0)$ & $0.527^{\mathrm{a}}$ \\
\hline
\end{tabular}

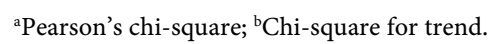


Finally, teachers who had their information about diabetes from the internet or health educator expressed more positive attitude towards the disease than their counterparts, $\mathrm{p}<0.05$.

\subsection{Diabetes-Related Practice}

Overall, good diabetes-related practice was observed among 54.3\% of teachers. The majority of teachers reported regular measuring of their weight (90.7\%) and height $(81.7 \%)$. Most teachers considered themselves non-smokers $(80.1 \%)$ as well as practice healthy eating habits (78.7\%). On the other hand, only less than half of the participants do not eat high calorie meals between main meals (47.2\%). Also, less than half of them reported measuring their blood pressure (43.1\%), blood cholesterol level (36.8\%) and blood glucose level (29.9\%).

Factors associated with diabetes-related practice are shown in Table 6. Participants' level of practice related to diabetes was significantly associated with their age. The highest level of good practice was among teachers aged 51 to 60 years (67.9\%), whereas the lowest level was among 20 to 30 year old teachers (38.8\%), $\mathrm{p}<0.001$. Principles had the highest level of good diabetes-related practice (86.7\%), while student counselors had the lowest $(42.3 \%), \mathrm{p}=0.002$. Almost two-thirds (66.2\%) of participants with teaching experience of more than 20 years expressed good diabetes-related practice as compared to only $34.8 \%$ of those with teaching experience of 1 to 5 years, $\mathrm{p}<0.001$. Non-Saudi teachers showed to have good diabetes related practice at a higher level than Saudi teachers $(66.7 \%$ versus $52.3 \%), \mathrm{p}=0.011$. Diabetic teachers had higher level of diabetes-related practice than non-diabetic teachers (69.6\% versus 51.1\%), $\mathrm{p}<0.001$. Also, all teachers that are diabetic for more than 20 years had good diabetes-related practice compared to $57.1 \%$ of teachers with less than one year history of DM, $\mathrm{p}=0.009$. Moreover, teachers with a family history of DM had good diabetes-related practice compared to teachers with no family history of DM (56.9\% versus $46.9 \%), \mathrm{p}$ $=0.028$. Finally, teachers who had their information about diabetes from medical book/scientific journal, health educator, physician or educational campaign had better disease-related practice than their counterparts, $\mathrm{p} \leq 0.001$.

\section{Discussion}

Most diabetes KAP studies done worldwide and in KSA focused on assessing diabetic patients, whereas limited studies were done to assess teachers despite their vital role in this regards. Therefore, this study was carried out to assess the KAP related to diabetes among teachers in Riyadh, KSA and to identify the associated factors.

Some of the participants' characteristics in this study were shared with the characteristics of teachers in Riyadh city as reported by the Department of Statistics at the Ministry of Education. The similarities were shown in the gender of teachers as almost half are males and the other half are females. Another shared characteristic was the type of school as around two-thirds of teachers work in public schools, while one-third of them work in private schools. 
Table 6. Factors associated with teachers' diabetes-related practice.

\begin{tabular}{|c|c|c|c|}
\hline \multirow[b]{2}{*}{ Variables } & \multicolumn{2}{|c|}{ Practice regarding Diabetes Mellitus } & \multirow[b]{2}{*}{ p-value } \\
\hline & $\begin{array}{c}\text { Poor }(\mathrm{N}=289) \\
\mathrm{N}(\%)\end{array}$ & $\begin{array}{c}\text { Good }(N=344) \\
N(\%)\end{array}$ & \\
\hline \multicolumn{4}{|l|}{ Age } \\
\hline $20-30(n=49)$ & $30(61.2)$ & $19(38.8)$ & \\
\hline $31-40(n=204)$ & $112(54.9)$ & $92(45.1)$ & \\
\hline $41-50(n=249)$ & $105(42.2)$ & $144(57.8)$ & \\
\hline $51-60(\mathrm{n}=131)$ & $42(32.1)$ & $89(67.9)$ & $<0.001^{\mathrm{b}}$ \\
\hline \multicolumn{4}{|l|}{ Gender } \\
\hline Male $(\mathrm{n}=331)$ & $156(47.1)$ & $175(52.9)$ & \\
\hline Female $(n=302)$ & $133(44.0)$ & $169(56.0)$ & $0.436^{\mathrm{a}}$ \\
\hline \multicolumn{4}{|l|}{ Qualification } \\
\hline Diploma $(n=42)$ & $15(35.7)$ & $27(64.3)$ & \\
\hline Bachelor $(\mathrm{n}=536)$ & $247(46.1)$ & $289(53.6)$ & \\
\hline Master $(\mathrm{n}=48)$ & $25(52.1)$ & $23(47.9)$ & \\
\hline $\mathrm{PhD}(\mathrm{n}=7)$ & $2(28.6)$ & $5(71.4)$ & $0.367^{\mathrm{b}}$ \\
\hline \multicolumn{4}{|l|}{ Major } \\
\hline Mathematics $(\mathrm{n}=75)$ & $37(49.3)$ & $38(50.7)$ & \\
\hline Science $(\mathrm{n}=79)$ & $31(39.2)$ & $48(60.8)$ & \\
\hline Islamic studies $(\mathrm{n}=134)$ & $58(43.3)$ & $76(56.7)$ & \\
\hline Art education $(n=45)$ & $23(51.1)$ & $22(48.9)$ & \\
\hline Social studies $(\mathrm{n}=83)$ & $48(57.8)$ & $35(42.2)$ & \\
\hline Arabic language $(\mathrm{n}=140)$ & $60(42.9)$ & $80(57.1)$ & \\
\hline English language $(\mathrm{n}=54)$ & $27(50.0)$ & $27(50.0)$ & \\
\hline Physical education $(\mathrm{n}=23)$ & $5(21.7)$ & $18(78.3)$ & $0.057^{\mathrm{a}}$ \\
\hline \multicolumn{4}{|l|}{ Professional title } \\
\hline Principal $(\mathrm{n}=30)$ & $4(13.3)$ & $26(86.7)$ & \\
\hline Deputy principal $(\mathrm{n}=33)$ & $14(42.4)$ & $19(57.6)$ & \\
\hline Teacher $(\mathrm{n}=544)$ & $256(47.1)$ & $288(52.9)$ & \\
\hline Student counselor $(\mathrm{n}=26)$ & $15(57.7)$ & $11(42.3)$ & $0.002^{\mathrm{a}}$ \\
\hline \multicolumn{4}{|l|}{ Type of school } \\
\hline Public $(\mathrm{n}=409)$ & $194(47.4)$ & $215(52.6)$ & \\
\hline Private $(n=224)$ & $95(42.4)$ & $129(57.6)$ & $0.225^{\mathrm{a}}$ \\
\hline \multicolumn{4}{|l|}{ Years of experience } \\
\hline $1-5(n=66)$ & $43(65.2)$ & $23(34.8)$ & \\
\hline $6-10(n=121)$ & $61(50.4)$ & $60(49.6)$ & \\
\hline $11-15(\mathrm{n}=79)$ & $43(54.4)$ & $36(45.6)$ & \\
\hline $16-20(\mathrm{n}=104)$ & $53(51.0)$ & $51(49.0)$ & \\
\hline$>20(\mathrm{n}=263)$ & $89(33.8)$ & $174(66.2)$ & $<0.001^{\mathrm{b}}$ \\
\hline \multicolumn{4}{|l|}{ Marital status } \\
\hline Single $(\mathrm{n}=71)$ & $38(53.5)$ & $33(46.5)$ & \\
\hline Married $(n=562)$ & $251(44.7)$ & $311(55.3)$ & $0.158^{\mathrm{a}}$ \\
\hline \multicolumn{4}{|l|}{ Nationality } \\
\hline Saudi $(\mathrm{n}=543)$ & $259(47.7)$ & $284(52.3)$ & \\
\hline Non-Saudi $(n=90)$ & $30(33.3)$ & $60(66.7)$ & $0.011^{\mathrm{a}}$ \\
\hline
\end{tabular}

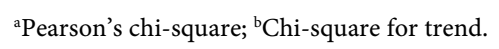


Also the majority of school employees were class teachers, meanwhile only $15 \%$ of them were administrative (principals, deputy principals, and student counselors) [17].

In the present study, knowledge of teachers about diabetes was sufficient in many aspects as more than half of them were described as knowledgeable. However, deficient knowledge was reported in some important aspects of the disease. Knowledge gaps were observed in the knowledge about fasting blood glucose cut-off level to diagnose diabetes, stroke as a complication of DM and smoking as a risk factor of it. In a similar Saudi study carried out in Al-Jouf region among primary and intermediate schools' teachers, adequate general knowledge about diabetes was also reported [2]. This sufficient general knowledge about diabetes could be attributed to the high prevalence of diabetes in the Saudi community, as well as the high educational level of the study population.

In Spain only $36.9 \%$ of the surveyed teachers had sufficient basic knowledge about diabetes, comparison between the studies is not applicable due to use of different tools and cut-off levels in assessing knowledge [5]. Nevertheless, other studies also strongly recommended a training program for teachers to increase their knowledge about diabetes after observing low to moderate levels of knowledge [19] [20].

Lower rates of knowledge were reported among the general population as compared to the more educated study population included in this study. For example, in Al-Khobar city in eastern KSA around $40 \%$ of primary healthcare center attendees were knowledgeable of diabetes risk factors and prevention. This lack of knowledge was attributed to the education status of the participants [23]. Another study was carried out to assess KAP among diabetic and non-diabetic patients from the outpatient department of two hospitals in Riyadh. The study revealed that $32 \%$ and $25 \%$ of diabetic and non-diabetic patients, respectively had good knowledge regarding diabetes [11].

Older more experienced teachers were more knowledgeable about diabetes than young teachers with less teaching experience. Also, more educated teachers expressed a higher level of knowledge than others. This finding is consistent with another study that showed an association between the level of education and the level of knowledge regarding diabetes [24].

The current study also revealed better knowledge about diabetes among science teachers than others. This finding is predicted due to the nature of their undergraduate major. In Jordan, a study included around 1700 diabetic patients reported that $53.5 \%$ of the participants had good knowledge about diabetes. Almost $40 \%$ of participants in that study had an undergraduate degree related to the medical field [21].

Male teachers had more knowledge about diabetes than female teachers in this study. We did not find similar findings in other studies done in KSA. However, this could be indorsed by the fact that in the Saudi population the prevalence of DM among males is higher than females [25]. 
In a previous study [12], comparing the knowledge about DM between diabetics and non-diabetic. The study found that diabetics had more knowledge than non-diabetics. The current study also found that diabetic teachers showed to have more knowledge than non-diabetic teachers. Another finding in this study, was teachers who have family members diagnosed with diabetes had a higher level of knowledge about the disease than teachers with no family history of diabetes. The previous finding was shared with a similar study conducted in Al-Jouf [2].

As observed in this study and in other studies carried out in KSA among the general population [11] [26], the role of health care professionals represented minimal contribution towards diabetes relevant knowledge. Thus, it is highly recommended to encourage health care professionals to have more active roles in raising the level of health related knowledge among the society.

In Riyadh, $56 \%$ and $48 \%$ of diabetic and non-diabetic patients, respectively expressed positive attitude towards diabetes [11]. In similarity to those figures, $53.1 \%$ of teachers in the current study showed to have positive attitude towards DM.

The study found that relatively more experienced teachers, diabetics with longer duration of the disease and those who had their information about diabetes from a health educator had more positive attitude towards DM than their counterparts. I believe that further research is needed to clarify these findings.

A study that was done in Turkey, surveyed 1500 teachers to assess their knowledge and attitude shared some findings with our study in regards of teachers' attitude [19]. The Turkish study found that around $90 \%$ of teachers were willing to accept diabetic students in their class. However, most of them reported feeling anxious when they were first informed that they have a diabetic child in their class [19]. In Al-Jouf Region, the high percentage of teachers willing to accept diabetic students in their class, meanwhile reported feeling anxious was also noticed [2]. This willing behavior of teachers to accept diabetic students, which was accompanied by anxiety is mainly attributed to mistrust in their knowledge about managing diabetic symptoms. This has been noticed in other studies that called out for a teacher educational or training program [19] [27].

In agreement with a study carried out in two hospitals in Riyadh city, that revealed $55 \%$ of diabetic patients and $52 \%$ of non-diabetic patients had good practice-related to diabetes [11]. The present study, found that $54.3 \%$ of teachers had good diabetes-related practice. This could be because of the wide spread of diabetes in the Saudi community, so good practices related to diabetes are admirable between all members of the society.

In this study the majority of teachers reported practicing healthy eating habits, being non-smokers, and regularly measuring their weight and height. The factors associated with good practice were older age, having longer teachers experience, working as a principal, having family history of diabetes, being diabetic, and having longer duration of DM. On the other hand, more than half of the participants eat high calorie meals between their main meals and do not measure their blood pressure, blood cholesterol or blood glucose level. 
Not surprisingly we found out that young teachers with less teaching experience had the least KAP level in all three domains. Because of that, targeting new young teachers should be of high priority in developing a diabetes education program for teachers. Moreover, new young teachers should be targeted because they are usually keener to learn, and they have a longer time before reaching their retirement age.

Knowing the source of the participants' information is useful in specifying the most appropriate method for health education to increase the KAP level of teachers. The most frequent sources of information about DM were relatives/friends, social media, internet, physician, and awareness campaign. Meanwhile, the highest level of KAP was shown in teachers who reported their source of information as medical book/scientific journal, health educator, and physician. This shows the importance of obtaining the health related information from scientific literature and healthcare professionals. Also this shows the significance of having a reliable trusted source of information for teachers, which will be beneficial in improving their KAP level as well as make teachers more confident in sharing and applying their KAP. Additionally, health care workers should be encouraged to participate in raising the level of teachers' KAP about diabetes.

Finally, focusing on increasing diabetes related KAP among teachers will help them lead healthy lifestyles. In addition, improve their role in supporting diabetic students at school. Therefore, there should be continuous education and counseling activities for Saudi teachers to encourage them to have a higher level of KAP.

\section{Conclusions}

Knowledge of teachers in public and private schools in Riyadh about diabetes is sufficient in many aspects. However, deficient knowledge was reported among some important aspects of the disease. Older and more experienced teachers were more knowledgeable about diabetes than younger and less experienced teachers. Teachers with a higher level of education showed to have better knowledge level than others. The current study showed that science teachers had a higher level of knowledge compared to others. Diabetics and teachers with a family history of DM were more knowledgeable about the disease. More than half of the teachers had positive attitude and good diabetes-related practice towards DM. Diabetics and teachers that reported to have a diabetic student, showed to have more positive attitude towards DM than their counterparts. Also Older, more experienced, non-Saudi, diabetic teachers with a longer duration of DM and with a family history of the disease showed the highest level of good diabetes-related.

Participants with more teaching experience showed a higher level across all aspects of KAP, as opposed to teachers with only 1 to 5 years of experience. So, targeting new young teachers should be a priority in order to raise their KAP level. The most frequent sources of information about DM were relatives/friends, social media, internet, physician and awareness campaign. Although, the highest level of KAP was among participants who reported medical book/scientific journal, 
health educator and physician as their source of information. Therefore, we can conclude that having a reliable trusted source of information about DM for teachers will be beneficial to improve their KAP level as well as make them more confident in sharing and applying their KAP. Finally, health care workers should be strongly encouraged to participate and play a role in raising the level of teachers' KAP about diabetes.

\section{Limitations}

This study is limited by the cross-sectional design, which proves association and not causality between dependent and independent variables. Another limitation was the low response rate, which made the study subjected to non-response bias. Therefore, participants' KAP level might be overestimated.

\section{Conflicts of Interest}

The author declares no conflicts of interest regarding the publication of this paper.

\section{References}

[1] The American Diabetes Association (2019) Classification and Diagnosis of Diabetes: Standards of Medical Care in Diabetes 2019. Diabetes Care, 42, S13-S28. https://doi.org/10.2337/dc19-S002

[2] Duraywish, N.A. (2017) Assessment of the Primary and Intermediate School Staffs' Knowledge, Attitude and Practice on Care of Children with Type 1 Diabetes at School. Sudan Journal of Medical Sciences, 12, 25-34.

https://doi.org/10.18502/sjms.v12i1.857

[3] Atlas, D. (2019) International Diabetes Federation. IDF Diabetes Atlas, 9th Edition, International Diabetes Federation, Brussels.

[4] Jackson, C.C., Albanese-O’Neill, A., Butler, K.L., Chiang, J.L., Deeb, L.C., Hathaway, K., et al. (2015) Diabetes Care in the School Setting: A Position Statement of the American Diabetes Association. Diabetes Care, 38, 1958-1963. https://doi.org/10.2337/dc15-1418

[5] Gutierrez-Manzanedo, J.V., Carral-San Laureano, F., Moreno-Vides, P., de Castro-Maqueda, G., Fernandez-Santos, J.R. and Ponce-Gonzalez, J.G. (2018) Teachers' Knowledge about Type 1 Diabetes in South of Spain Public Schools. Diabetes Research and Clinical Practice, 143, 140-145. https://doi.org/10.1016/j.diabres.2018.07.013

[6] Deylami, R., Townson, J., Mann, M. and Gregory, J.W. (2018) Systematic Review of Publicity Interventions to Increase Awareness amongst Healthcare Professionals and the Public to Promote Earlier Diagnosis of Type 1 Diabetes in Children and Young People. Pediatric Diabetes, 19, 566-573. https://doi.org/10.1111/pedi.12565

[7] Al-Nozha, M.M., Al-Maatouq, M.A., Al-Mazrou, Y.Y., Al-Harthi, S.S., Arafah, M.R., Khalil, M.Z., et al. (2004) Diabetes Mellitus in Saudi Arabia. Saudi Medical Journal, 25, 1603-1610.

[8] Al-Rubeaan, K., Al-Manaa, H.A., Khoja, T.A., Ahmad, N.A., Al-Sharqawi, A.H., Siddiqui, K., et al. (2015) Epidemiology of Abnormal Glucose Metabolism in a Country Facing Its Epidemic: SAUDI-DM Study. Journal of Diabetes, 7, 622-632. https://doi.org/10.1111/1753-0407.12224 
[9] Alhowaish, A.K. (2013) Economic Costs of Diabetes in Saudi Arabia. Journal of Family \& Community Medicine, 20, 1-7. https://doi.org/10.4103/2230-8229.108174

[10] Bowman, B.A., Gregg, E.W., Williams, D.E., Engelgau, M.M. and Jack Jr., L. (2003) Translating the Science of Primary, Secondary, and Tertiary Prevention to Inform the Public Health Response to Diabetes. Journal of Public Health Management and Practice, S8-S14. https://doi.org/10.1097/00124784-200311001-00002

[11] Salem, A., Majed, A., Mahfooz Mustafa, M., Abdulsalam, A., Mohammed Basheeruddin Asdaq, S. and Mohammed, A.Y. (2018) Knowledge, Attitude, and Practice Regarding Diabetes Mellitus among General Public and Diabetic Patients in Riyadh, Saudi Arabia. Asian Journal of Pharmaceutics, 12, S268-S276.

[12] Alanazi, F.K., Alotaibi, J.S., Paliadelis, P., Alqarawi, N., Alsharari, A. and Albagawi, B. (2018) Knowledge and Awareness of Diabetes Mellitus and Its Risk Factors in Saudi Arabia. Saudi Medical Journal, 39, 981-989. https://doi.org/10.15537/smj.2018.10.22938

[13] Alzahrani, M.A.A. (2019) Teachers' Knowledge of Diabetes and Attitudes towards Diabetic Students in the Primary Schools in Al Baha City in Saudi Arabia. International Journal of Education and Literacy Studies, 7, 156-171. https://doi.org/10.7575/aiac.ijels.v.7n.2p.156

[14] Mohammed Almehmad, R., Bin Qadir, S.A., Mohammed Taweel, K., Abdulkarim Marouf, M., Hasan Algarni, A. and Mohamad Qadah, B. (2018) Awareness of School Teachers about Diabetes Mellitus. Egyptian Journal of Hospital Medicine, 70, 1230-1233. https://doi.org/10.12816/0044555

[15] Abahussain, N.A. and El-Zubier, A.G. (2005) Diabetes Knowledge among Self Reported Diabetic Female Teachers: Al-Khobar, Saudi Arabia. Journal of Family \& Community Medicine, 12, 43-48.

[16] (2016) Population Study of Riyadh City (1437H). Royal Commission for Riyadh City, Riyadh.

http://www.ada.gov.sa/res/ada/ar/Researches/Riyadh-Population-Study-1437H-201 6/index.html

[17] Ministry of Education (2016-2017) General Summary: Schools, Classes, Students, Faculty, Assistant Administrators, and Full-Time Employees According to the Stages and Types of Education in Boys and Girls Schools in the Kingdom. Riyadh. https://edu.moe.gov.sa/Riyadh/DocumentCentre/Pages/default.aspx?.DocId=c5533d a3-5962-4000-8737-da6749bdfe2a

[18] OpenEpi (2019) Open Source Statistics for Public Health: OpenEpi, Version 3. https://www.openepi.com/SampleSize/SSPropor.htm

[19] Aycan, Z., Önder, A., Çetinkaya, S., Bilgili, H., Yıldırım, N., Baş, V.N., et al. (2012) Assessment of the Knowledge of Diabetes Mellitus among School Teachers within the Scope of the Managing Diabetes at School Program. Journal of Clinical Research in Pediatric Endocrinology, 4, 199-203. https://doi.org/10.4274/Jcrpe.756

[20] Taha, N.A., Rahme, Z., Mesbah, N., Mahmoud, F., AlKandari, S., Othman, N., et al. (2018) Evaluation of the Impact of a Diabetes Education e-Learning Program for School Personnel on Diabetes Knowledge, Knowledge Retention and Confidence in Caring for Students with Diabetes. Diabetes Research and Clinical Practice, 139, 348-356. https://doi.org/10.1016/j.diabres.2018.03.019

[21] Alsous, M., Abdel Jalil, M., Odeh, M., Al Kurdi, R. and Alnan, M. (2019) Public Knowledge, Attitudes and Practices toward Diabetes Mellitus: A Cross-Sectional Study from Jordan. PLOS ONE, 14, e0214479.

https://doi.org/10.1371/journal.pone.0214479 
[22] Kassahun, C.W. and Mekonen, A.G. (2017) Knowledge, Attitude, Practices and Their Associated Factors towards Diabetes Mellitus among Non Diabetes Community Members of Bale Zone Administrative Towns, South East Ethiopia. A Cross-Sectional Study. PLoS ONE, 12, e0170040.

https://doi.org/10.1371/journal.pone.0170040

[23] Aljoudi, A.S. and Taha, A.Z. (2009) Knowledge of Diabetes Risk Factors and Preventive Measures among Attendees of a Primary Care Center in Eastern Saudi Arabia. Annals of Saudi Medicine, 29, 15-19. https://doi.org/10.4103/0256-4947.51813

[24] Kamel, N.M., Badawy, Y.A., el-Zeiny, N.A. and Merdan, I.A. (1999) Sociodemographic Determinants of Management Behaviour of Diabetic Patients. Part II. Diabetics' Knowledge of the Disease and Their Management Behaviour. Eastern Mediterranean Health Journal, 5, 974-983.

[25] Alqurashi, K.A., Aljabri, K.S. and Bokhari, S.A. (2011) Prevalence of Diabetes Mellitus in a Saudi Community. Annals of Saudi Medicine, 31, 19-23. https://doi.org/10.4103/0256-4947.75773

[26] Sabra, A.A., Taha, A.Z., Al-Zubier, A.G. and Al-Kurashi, N.Y. (2010) Misconceptions about Diabetes Mellitus among Adult Male Attendees of Primary Health Care Centres in Eastern Saudi Arabia. South African Family Practice, 52, 344-349. https://doi.org/10.1080/20786204.2010.10874004

[27] Alnasir, F.A. (2003) Assessment of Knowledge of Diabetes Mellitus among Bahraini School Teachers. Bahrain Medical Bulletin, 25, 172-176. 


\section{CONSENT FORM}

تقييم المعرفه والسلوك والممارسات المتعلقة بمرض السكري عذ المعلمين والمعلمات في مدينة الرياض في المملكة العربية السعودية

An Assessment of the Diabetic Knowledge, Attitude, and Practice of School Teachers in Riyadh, Kingdom of Saudi Arabia.

Principal Investigator: Dr. Ghassan Aldekhayel

You are being asked to participate voluntarily in a research by answering this questionnaire. If you decide to take part in this study, please sign this consent form.

STUDY PURPOSE: To assess the knowledge, attitude, and practice about type 2 diabetes among school teachers in Riyadh, Saudi Arabia.

BENEFITS: The result of this study may help decision makers to develop policies that will enhance the health care system in the Kingdom of Saudi Arabia.

SIDE EFFECT: There are no side effects. Your participation in this study does not have any further risks or discomfort to you.

REFUSAL: If you refuse to participate, there will be no penalty or loss of benefits.

CONFIDENTALITY: Your participation in this study will be kept confidential. The results of this research may be published; however, your identity will never be revealed.

APPROVAL: I fully understand the information and the consent form.

I sign freely and voluntarily.

Signature:

Date:

This questionnaire may take from 5-7 minutes.

If you have any further concerns or questions, you can contact Dr. Ghassan Aldekhayel.

(Email: Dr.gass@hotmail.com)

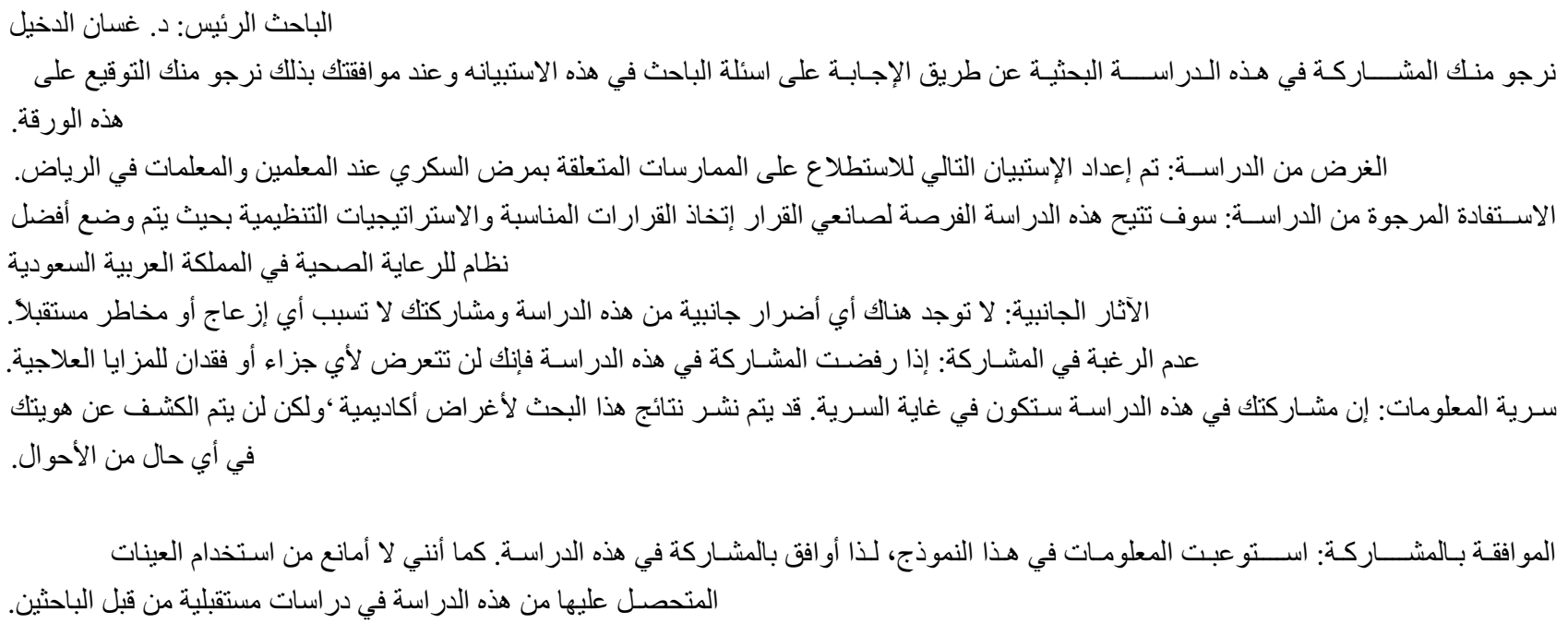




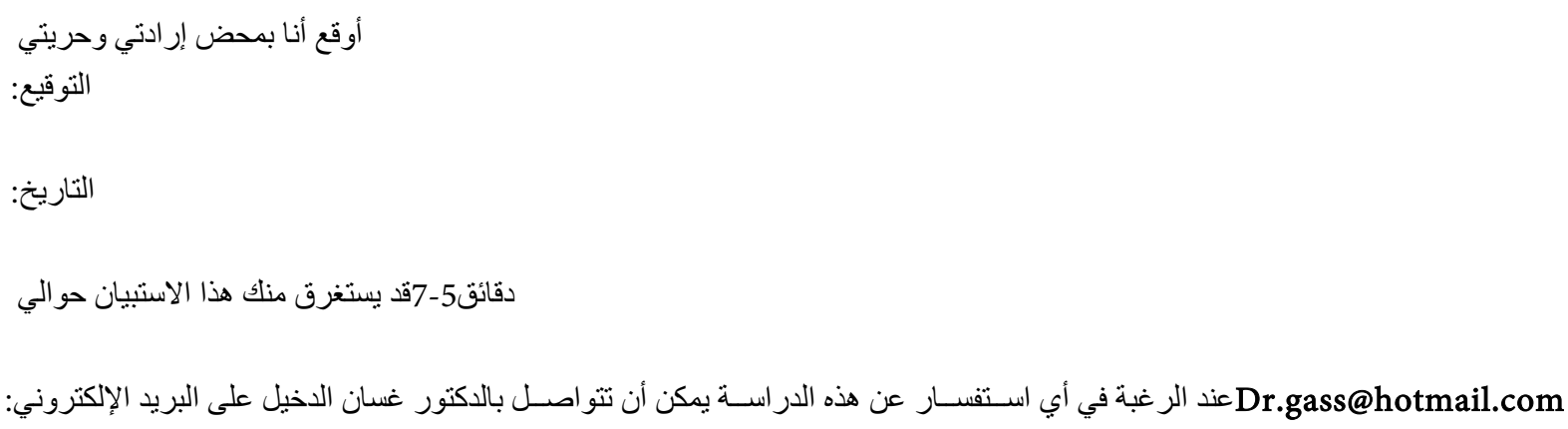

\section{Part One: Personal Details}

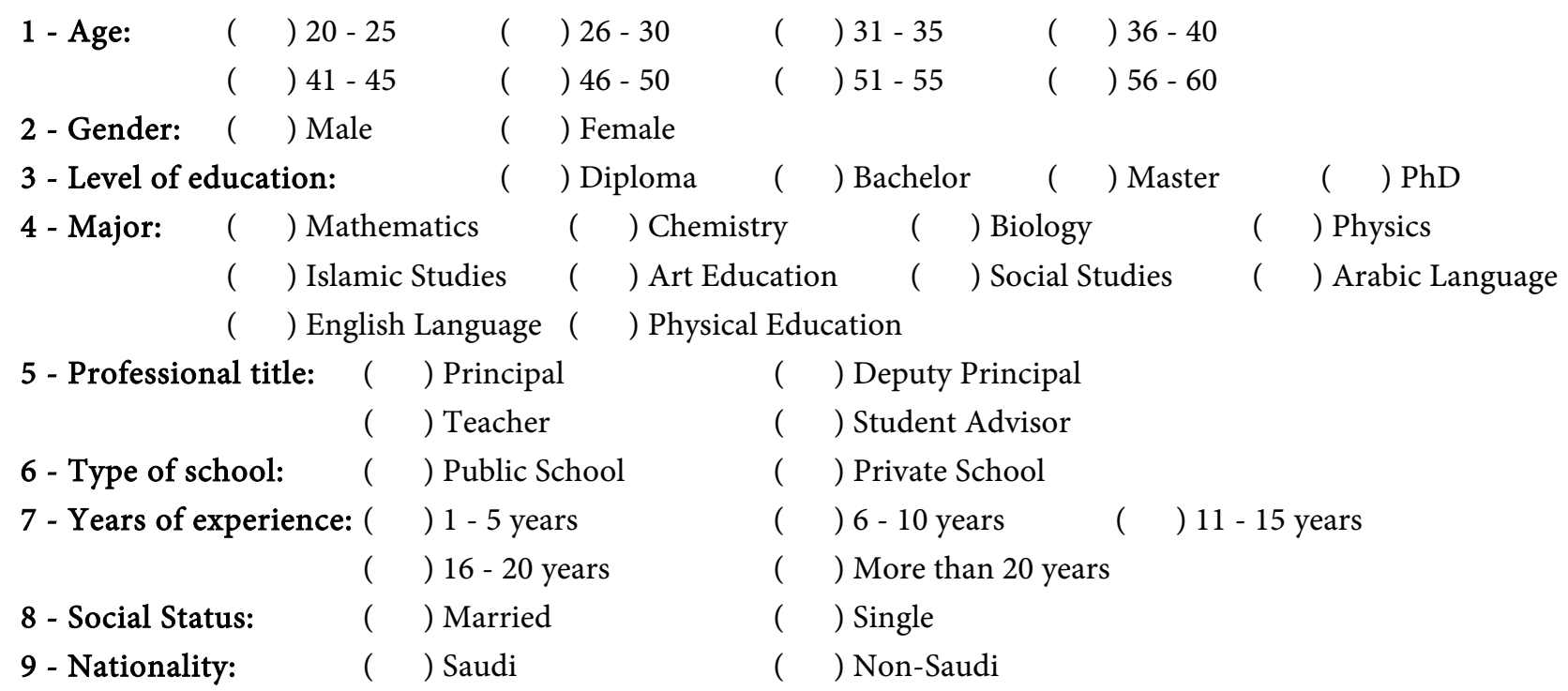

\section{Part Two: Knowledge}

1 - Source of information about diabetes (more than one answer can be selected)

\begin{tabular}{|c|c|c|}
\hline ) Relative/friend & ( ) Social media & ( ) Medical book/scientific journal \\
\hline ) Search on the Internet & ( ) Health educator & ( ) Physician \\
\hline
\end{tabular}

2 - Do you suffer from diabetes? ( ) Yes ( ) No

3 - If the answer is Yes to the previous question, How long have you suffered from diabetes?
( ) less than one year
( ) $1-5$ years
( ) 6 - 10 years
( ) $11-15$ years
( ) $16-20$ years
( ) More than 20 years

4 - Do you have anyone in the family with diabetes? ( ) Yes ( ) No

5 - If the answer is Yes to the previous question, What is your relationship to him/her?
( ) Parents
( ) Spouse
( ) Brother/Sister
( ) Son/Daughter

6 - A person is diagnosed with diabetes if the measurement of blood sugar after fasting is more than?
( ) $116 \mathrm{mg} / \mathrm{dl}$
) $126 \mathrm{mg} / \mathrm{dl}$
( ) $146 \mathrm{mg} / \mathrm{dl}$
( ) Don't Know

7 - Which of the following organs dysfunction leads to diabetes?
( ) Pancreas
) Stomach
( ) Kidney
( ) Liver
( ) Don't Know 
8 - Diabetes can cause: (more than one answer can be selected)
( ) Brain stroke
( ) Heart attack
( ) Hepatitis
( ) Kidney failure
( ) Arthritis
( ) Retinopathy
( ) Neuropathy
( ) Don't Know

9 - Which of the following increase the risk of diabetes: (more than one answer can be selected)
( ) Obesity
( ) Eating fast food
( ) Genetic factors
Stress
( ) Eating carbohydrate
( ) Smoking
( ) Sitting for long hours
( ) Don't Know

10 - Do you know that diabetes can be prevented through sports and eating healthy?
( ) Yes
( ) No
( ) Don't Know

11 - Does the school have a glucometer?
( ) Yes
( ) No
( ) Don't Know

12 - Do you have any student with diabetes in your class?
( ) Yes
( ) No
) Don't Know

\section{Part Three: Attitude}

1 - Do you think that sports are better than taking prescription medications to treat diabetes?
( ) Yes
( ) No
( ) Don't Know

2 - Do you think that the use of alternative medicine such as herbs (ginger, cinnamon and fenugreek) is better than taking medications prescribed by a physician to treat diabetes?
( ) Yes
( ) No
( ) Don't Know

3 - Do you think it is useless to try to control blood glucose because complications of diabetes will occur anyway?
( ) Yes
( ) No
( ) Don't Know

4 - Do you think that attending awareness and educational lectures about diabetes will help improve a diabetic patient?
( ) Yes
( ) No
( ) Don't Know

5 - Did you feel anxious when you were first informed that you have children with diabetes in your class?
( ) Yes
( ) No
( ) Don't Know

6 - Are you willing to accept diabetic students in your class?
( ) Yes
( ) No
( ) Don't Know

7 - Does the use of insulin lead to the addiction of the body to insulin?
( ) Yes
( ) No
( ) Don't Know

\section{Part Four: Practice}

1 - Do you practice healthy eating habits in your lifestyle? (Like eating salads)
( ) Yes
( ) No

2 - Do you exercise daily for half an hour or more? (Such as sports, brisk walking, household activities, stair climbing)
( ) Yes
( ) No

3 - Do you eat high calorie meals between your main meals? (Like carbohydrate and fat)?
( ) Yes
( ) No

4 - Do you practice the habit of eating junk food at least once a week? (Such as hamburger, pizza, pastries, shawarma, French fries)
( ) Yes
( ) No 
5 - If a diabetic student fainted and a glucometer was not available, what would you do first?

( ) Take the student to the hospital

( ) Put sugar or juice in the student's mouth

( ) Contact the student's parent

( ) Give the student a dose of insulin

6 - A diabetic student comes to you and says "I feel weak", what would you do first?

( ) Take the student to the hospital

( ) Give the student some sugar, or a small bottle of juice

( ) Contact the student's parent

( ) Give the student a dose of insulin

7 - Are diabetic children eligible to attend physical education classes?
( ) Yes
( ) No
( ) Don't Know

8 - How do you classify yourself regarding the habit of smoking? (Cigarettes, hookahs)

( ) Currently non-smoker ( ) Former smoker ( ) Smoker at social events

( ) A smoker who wants to quit smoking

( ) A smoker who wants to minimize smoking

( ) A smoker who wants to continue smoking

9 - Do you keep your body fit?

10 - Do you eat your main meals at a specific time?

11 - Do you measure your blood glucose level?
( ) Yes
( ) No
What is your blood glucose level $(\mathrm{mg} / \mathrm{dl})$ :

12 - Do you measure your blood pressure level?

$($ ) Yes ( ) No Blood pressure level: $\quad(\quad)$ Normal $\quad(\quad)$ High

13 - Do you measure your cholesterol level?

( ) Yes ( ) No Cholesterol level:

14 - Do you measure your weight?

( ) Yes

( ) Yes

( ) No

( ) Yes

( ) No

15 - Do you measure your height?

( ) Yes

( ) Normal ( ) High

( ) No What is your weight $(\mathrm{Kg}): \ldots \ldots$...

( ) No

What is your height $(\mathrm{cm}): \ldots \ldots$.

\section{(Optional)}

If you are interested in checking your blood glucose level, please fill in your name and number to contact you

Name:

Mobile Number: 